DIFFERENCE VERSUS GAUSSIAN CURVATURE ENERGIES; MONOLAYER VERSUS BILAYER CURVATURE ENERGIES APPLICATIONS TO VESICLE STABILITY

$$
\text { By }
$$

Håkan Wennerström

and

David M. Anderson

IMA Preprint Series \# 910

December 1991 


\title{
DIFFERENCE VERSUS GAUSSIAN CURVATURE ENERGIES; MONOLAYER VERSUS BILAYER CURVATURE ENERGIES APPLICATIONS TO VESICLE STABILITY
}

\author{
HÅAN WENNERSTRÖM* AND DAVID M. ANDERSON*†
}

\begin{abstract}
The recent discovery that the electrostatic contribution to the curvature free energy $G_{c}$ of a membrane has, exactly to second order in the principle curvatures $c_{2}$ and $c_{1}$, the form $G_{c}^{E}=b^{E} \iint\left(c_{2}-\right.$ $\left.c_{1}\right)^{2} d A$ [B. Duplantier, Physica A, 168, 179-197, eqn. 39] is found to be symptomatic of the group theoretic properties of $G_{c}\left(c_{1}, c_{2}\right)$. These properties, together with the need to re-instate stability criteria into a positive-definite expression for $G_{c}$ and to single out monolayer mean curvature energy as the predominant contribution - rather than writing it as the difference between mean and Gaussian terms of comparable magnitude - all attest that the curvature free energy is most appropriately derived by expanding the free energy density $g_{c}$ directly in the principal curvatures $c_{1}, c_{2}$, rather than in the mean and Gaussian curvatures $H, K$. Using symmetry-adapted expansion coordinates we find to second order $g_{c}=a H^{2}+b \Delta c^{2}$ (where $2 H=c_{1}+c_{2} ; 2 \Delta c=c_{2}-c_{1}$ ) for a system of ground state $D_{\infty}\left(D_{\infty h}\right)$ symmetry, i.e. a symmetric bilayer, where the curvatures $c_{1}$ and $c_{2}$ are measured on the bilayer midsurface. For a system of $C_{\infty}$ (or $C_{\infty h}$ ) symmetry, we find $g_{c}=a\left(H-c_{0}\right)^{2}+b \Delta c^{2}$; this expression applies to both the asymmetric bilayer, in which $c_{1}$ and $c_{2}$ are measured on the bilayer midsurface, and to the more general case of a (paired or unpaired) surfactant monolayer, where they are registered at the polar-apolar interface. The last formalism most directly incorporates experimental observables.

With this expression, $b \ll a$ inherently, making it clear that not two but one rigidity constant is needed to interpret phase progressions which are driven by mean curvature. Indeed, recent work on bicontinuous $L_{3}$ phases and on progressions between cubic phases of different symmetries extends the time-tested notion that patterns in phase behavior over ranges of solvents, surfactants, etc. can be simply and quantitatively modeled with one mean curvature energy term. As a further demonstration of the transparency of this approach, using this expansion $g_{c}(H, \Delta c)$, the value of $b$ is independent of $c_{0}$ and non-negative - e.g., it does not change sign as $c_{0}$ or $H_{0}$ vary - and should be more easily measurable than is a rigidity "constant" which is expected to change (even its sign) with composition and temperature. Furthermore, $c_{0}\left(\neq H_{0}\right.$ in general) represents the optimal mean curvature in an unconstrained system; this is not the case with the $H, K$ expansion, which is thus once again seen to be distanced from the experimental intuition and interpretation. The expansion of $g_{c}$ in terms of $H$ and $\Delta c$ also clearly reveals the presence of two types of bending fluctuation modes.

These expressions are then used to discuss the thermodynamic stability of spherical vesicles from a curvature energy point of view. The expansions to second, and even to fourth, order are greatly simplified compared to the $H, K$ formalism because the "difference curvature" is rigorously zero and cross terms do not enter. It is derived that single-component vesicles can only be stable with a rather special combination of elastic constants and the phenomenon should be observed only rarely. With two or more components in the vesicle there is an extra degree of freedom involving a composition difference between the center and inner monolayers, which makes vesicle stability a much more likely event.
\end{abstract}

*Department of Physical Chemistry 1, Chemical Center, University of Lund, P.O. Box 124, S-221 00 Lund, SWEDEN.

†Permanent address for DA: Biomaterials Dept., SUNY at Buffalo, Buffalo, NY 14214. 
Introduction. In order to evaluate the curvature free energy of a membrane, we must begin by constructing a mathematical surface and ascribing to it a certain symmetry, which reflects the local symmetry of the membrane. If the membrane is an unpaired monolayer, then this surface is most appropriately drawn at the locus of the bonds linking the polar and apolar portions of the amphiphilic molecules, and there is then an obvious asymmetry with respect to the two sides of the surface. If the membrane is a bilayer, then there are two choices. The bilayer can be viewed as two paired monolayers, and two surfaces drawn, each as in the unpaired monolayer case. Alternatively, the surface can be taken to be the midsurface of the bilayer, which essentially corresponds to the terminal methyl groups on the hydrophobic tails. If the bilayer is inherently symmetric with respect to its two monolayers, then this surface should be described as symmetric with respect to its two sides. This is commonly the case for model bilayers. However, in biological systems, such as cell membranes, additional components such as proteins, glycolipids, and macromolecules effect a natural asymmetry with respect to the two sides of the bilayer, and thus of the mathematical surface.

Given this surface and its ascribed local symmetry, the curvature free energy density is usually expressed ${ }^{1}$ in terms of the mean curvature ${ }^{2}$

$$
H=1 / 2\left(c_{1}+c_{2}\right)
$$

and the Gaussian curvature

$$
K=c_{1} c_{2}
$$

where $c_{1}$ and $c_{2}$ are the two principal curvatures. For a system of inherent $D_{\infty}\left(D_{\infty H}\right)$ symmetry, i.e., in-plane rotational symmetry characteristic of a two-dimensional fluid state and two-fold rotational axis in the membrane plane, as in a symmetric bilayer, the curvature free energy density $g_{c}$ is written as ${ }^{1,3}$

$$
g_{c}=\kappa H^{2}+\bar{\kappa} K
$$

where $\kappa$ is called the bending rigidity and $\bar{\kappa}$ the saddle splay constant. The total curvature free energy $G_{c}$ is obtained by integrating $g_{c}$ over the surface

$$
G_{c}=\int_{A} g_{c} d A=\kappa \int_{A} H^{2} d A+\bar{\kappa} \int_{A} K d A
$$

The expression (3) contains all the curvature terms to second order in the given symmetry. However, the choice of $H$ and $K$ as coordinates for characterizing the curvature properties is not unique and other linear combinations of terms second order in $c_{1}$ and $c_{2}$ could also be used. The rationale for choosing $H$ and $K$ is, to our knowledge, two-fold: i) it confirms 
with the elastic free energy expression used for nematic liquid crystals; and ii) it has the virtue of explicitly separating out a Gaussian curvature term, which in the integral of eq. (4) has a remarkably simple property due to the Gauss-Bonnet theorem ${ }^{4}$

$$
\int_{A} K d A=2 \pi \chi_{E}
$$

where $\chi_{E}$ is the Euler characteristic, which only depends on the topology of the surface (we have left out the boundary term for simplicity).

For membranes where there is no perpendicular twofold symmetry axis, like a surfactant monolayer or an asymmetric bilayer, the local symmetry is $C_{\infty}$ (or $C_{\infty h}$ ). Due to the lower symmetry an additional parameter is needed in the free energy density expression and conventionally

$$
g_{c}=\kappa\left(H-H_{0}\right)^{2}+\bar{\kappa} K
$$

where $H_{0}$ is called the spontaneous mean curvature.

The description of curvature energies in terms of eqs. (3) and (6) has a long tradition in biological membrane works, particularly for the red blood cell problem ${ }^{5}$. It has also been applied extensively to model membrane problems, particularly by Helfrich and coworkers $^{6-8}$. In these treatments, the surface on which the curvatures is measured, and over which the area integration is performed, is the midsurface of the bilayer. More recently the same concepts have been used in the description of microemulsions ${ }^{9}$, where the amphiphile is in the form of an unpaired monolayer and the appropriate surface is drawn within the monolayer.

There is also an independent tradition of using basically similar notions in rationalizing the rich phase behaviour of surfactant systems. The basic work in this respect goes back on one hand to $\operatorname{Tartar}^{10}$, Tanford ${ }^{11}$ and Israelachvili and coworkers ${ }^{12-14}$, who have stressed the molecular shape and the packing constraints imposed by it. On the other hand Winsor ${ }^{15}$ introduced so called $R$ values describing the preferred average radius of an aggregate. Using this concept he was able to rationalize the phase behavior by assuming regular changes in the $R$-value. In both approaches the focus is on the surfactant monolayer as the natural unit rather than a bilayer.

That monolayer mean curvature, whether expressed in terms of an $R$-value or $v / a_{0} L_{0}$ or $H$ measured at the polar-apolar interface, is dominant over other curvature energy terms designed to include curvature inhomogeneities is made clear not only by decades of surfactant phase behavior study, but also under more general conditions by more recent experimental and theoretical work. Phase behavior work by Tiddy and coworkers ${ }^{16}$, as well as by Auvray et al. ${ }^{17}$ has shown that the same patterns of phase behavior seen with water are observed over a wide variety of non-aqueous polar solvents, including aprotic, nonhydrogen bonding solvents such as $N$-methylsydnone. The phase behavior of bicontinuous 
microstructures such as the $L_{3}$ phase ${ }^{18}$ and bicontinuous cubic phases ${ }^{19}$ has been shown to be readily interpretted in terms of monolayer mean curvature. The latter work shows that simple mean curvature arguments can yield quantitative predictions of phase behavior and structural details, including progressions between cubic phases of different microstructure, without the need for Gaussian curvature terms. A cursory examination of phase behavior is enough to reveal that Gaussian curvature is not behaving even remotely monotonic in these systems: the Gaussian curvature in the familiar lamellar to cubic to hexagonal phase progression passes from zero to negative to zero, for example.

In our opinion there is much to be gained by trying to unify the more chemicallyoriented approaches with the curvature free energy approach. They rely on basically the same physical effects and one can take advantage of the particular strengths of each of the different approaches. This is one of the main aims of the present paper. We start by examining alternative second order expressions for $g_{c}$, which leads to the introduction of a "difference curvature" term. Then we show how the curvature energy of a bilayer is expressed in terms of the monolayer constants. They we discuss the experience gained from the surfactant systems to obtain qualitative estimates of the elastic constants and to support the notion that curvature energies are from a physical point of view best associated with the polar-apolar interface. The paper is concluded by an investigation of the thermodynamical stability of vesicles demonstrating the usefulness of the conceptual framework developed earlier in the paper.

Curvature Free Energy. The Difference Curvature. Let us derive the curvature free energy in a way slightly different from the usual one based on bulk continuum mechanics concepts. Assume that the total curvature free energy is given as in eq. (4) by an integral of curvature free energy density $g_{c}\left(c_{1}, c_{2}\right)$ being a unique function of local principal curvatures. In such an expansion it is convenient to choose symmetry adapted curvature coordinates, and only energy terms that transform as the totally symmetric representation of the particular symmetry group $\left(D_{\infty}\right.$ or $\left.D_{\infty h}\right)$ are non-zero. These coordinates are the mean curvature $H$, and the difference curvature

$$
\Delta c=\frac{1}{2}\left(c_{2}-c_{1}\right) ; \quad \Delta c^{2}=H^{2}-K
$$

In $D_{\infty}\left(D_{\infty h}\right)$ symmetry, $H$ transform as the $\sigma_{-}$and $\Delta c$ as the $\delta$ irreducible representation and

$$
g_{c}\left(c_{1}, c_{2}\right)=a H^{2}+b \Delta c^{2}
$$

to second order. Both first derivatives and the second order cross term vanish by symmetry. The elastic constants $a$ and $b$ are related to the bending rigidity and the saddle splay constant through

$$
\kappa=(a+b) / 2 ; \quad \bar{\kappa}=-b
$$


which is obtained by identifying eqs. (3) and (8). Note the mathematically trivial but conceptually nontrivial result that the coefficients $\kappa$ and $a$ for the mean curvature term are different. $\kappa$ measures the resistance to bending along one axes leaving the other direction flat, a locally cylindrical deformation, while $a$ measures the resistance to bending which is symmetrical in both directions, i.e., a locally spherical deformation.

In the lower symmetry, $C_{\infty}, H$ transforms as the totally symmetric representation and the first derivative $d g_{c} / d H$ is non-zero. It is then natural to expand $g_{c}$ around a point $\left\{H=c_{0}, \Delta c=0\right\}$ where the first derivatives are zero. Then, to second order for example

$$
g_{c}=a\left(H-c_{0}\right)^{2}+b \Delta c^{2}
$$

analogously to eq. (6). The relation between $a$ and $b$ on one hand and $\kappa$ and $\bar{\kappa}$ on the other is formally the same as in eq. $(9 a, b)$. However, while $a$ and $b$ are obtained as second order derivatives of $g_{c}$ around a specified point the coefficients in eq. (6) are phenomenological and thus more difficult to relate to a molecular theory of the same system. The preferred mean curvature $c_{0}$ is related to the spontaneous curvature through

$$
H_{0}=c_{0} a /(a+b)
$$

It is our impression that it is a common misconception to take $H_{0}$ as the optimal mean curvature of a topologically unconstrained system. For example, an unconstrained membrane system with $a, b>0$ would optimally form spheres of radius $\left|1 / c_{0}\right|$ rather than $\left|1 / H_{0}\right|$. The treatment of ref. 18 , as well as other theories which do not include Gaussian curvature terms and focus on the mean curvature, can be viewed in the present context as the expression (10) in which one takes $b \ll a$. Thus in cases where the mean curvature is taken to be the primary effect, the expansion (10) provides the framework to reduce the number of terms from two to one, whereas this cannot be done with expansion (4).

The authors take it as self-evident that there are clear advantages to formulating the curvature free energy expansion in such a way that all terms are non-negative and minimized at $H=c_{0}, \Delta c=0$.

Recently Duplantier ${ }^{20}$ has derived an exact solution of the electrostatic contribution to the curvature free energy expansion (taking the thickness of the bilayer to be neglible). The intent of that work was to write the solution in terms of the conventional $H, K$ expansion. However, in eq. 39 of that paper it shown that the most succinct way to write the result - viz., with one term instead of two - is as a difference curvature energy. This result holds for his cases a) and b), the conducting membrane and the transparent insulating membrane, resp. For case c), the opaque insulating membrane, the result can be written as the sum of an $H^{2}$ term and a difference curvature energy, conforming to our eq. (8).

It is our conclusion that many calculations and discussions using curvature free energy concepts, particularly including the effects of fluctuations, would become more lucid if they were based on the expansion eqs. (8) and (10) rather than on eqs. (3) and (6). The 
confusion caused by writing $H_{0}$ instead of $c_{0}$ provides one example, but probably the most important aspect of eqs. (8) and (10) is that they clearly display the presence of two different types of curvature fluctuation modes; those involving $H$ and those involving $\Delta c$. The latter, softer modes have received very little explicit attention in previous work. Implicitly they are responsible for the Talmon-Prager entropy of a bicontinuous microstructure. The simplifications offered by eqs. (8) and (10) are also apparent when one is considering spherical aggregates like microemulsion droplets or vesicles. In that case $\Delta c=0$ and the inclusion of the second term in eq. (10) is trivial. One case when eq. (3) may be preferred is when there is a physical constraint only allowing variations preserving the topology of the surface, as, for example, in the classical problem of the shape of erythrocytes. In such a case the Gaussian curvature term is a constant when integrated over the surface as shown in eq. (5).

Monolayer Versus Bilayer Curvature Energies. In biological and model membranes as well as in many surfactant systems, two amphiphile monolayers unite to form a bilayer as the local structural unit. However, this local unit can fold in different ways to give rise to various global structures, the most common being planar bilayers, vesicles, cubic bicontinuous phases and the $L_{3}$ or anomalous phase. In a recent paper ${ }^{18}$ we demonstrated how curvature concepts were very useful for rationalizing the peculiar properties of this latter phase. One key argument in the paper was that the curvature energies are best described in terms of those of the constituent monolayers. The same approach was used in a treatment of curvature free energies in inverted hexagonal phases ${ }^{21}$ and in bicontinuous cubic phases ${ }^{19,22}$. The physical assumption being, amply supported by the experience gained from surfactant systems in general, that the curvature energies are best associated with the polay-apolar interface.

The surfactant/water inverted hexagonal phase provides one example where the monolayer curvature approach is clearly advantageous. Difficulties arise if the structure is viewed in terms of bilayers, as is commonly done in the closely related lamellar and bicontinuous cubic phases. In the inverted hexagonal phase the midsurface of the bilayer is an unorientable surface, a honeycomb arrangement of flat strips which intersect at $120^{\circ}$ angles. Mean curvature singularities make approaches using eqn. (3) intractable. On the other hand, the polar-apolar interface of each monolayer is simply a circular (approximately) cylinder, and its mean curvature is uniform and, as is well-known, intermediate between that of the inverted cubic and the inverted micellar solution.

Figure 1 shows a cross section of a bilayer with a midsurface, or "base" surface, at the center and two displaced surfaces assumed to represent the polay-apolar interface. Below we will assume, for simplicity, that the displaced surfaces are parallel to the base surface at a distance $L$. The argument could equally well be persued for displaced surfaces of constant mean curvature ${ }^{18,22}$. For bilayers curving only moderately $(|H| \ll 1 / L)$ the difference between the two cases is small, while for highly curved cases the distinction becomes important. 


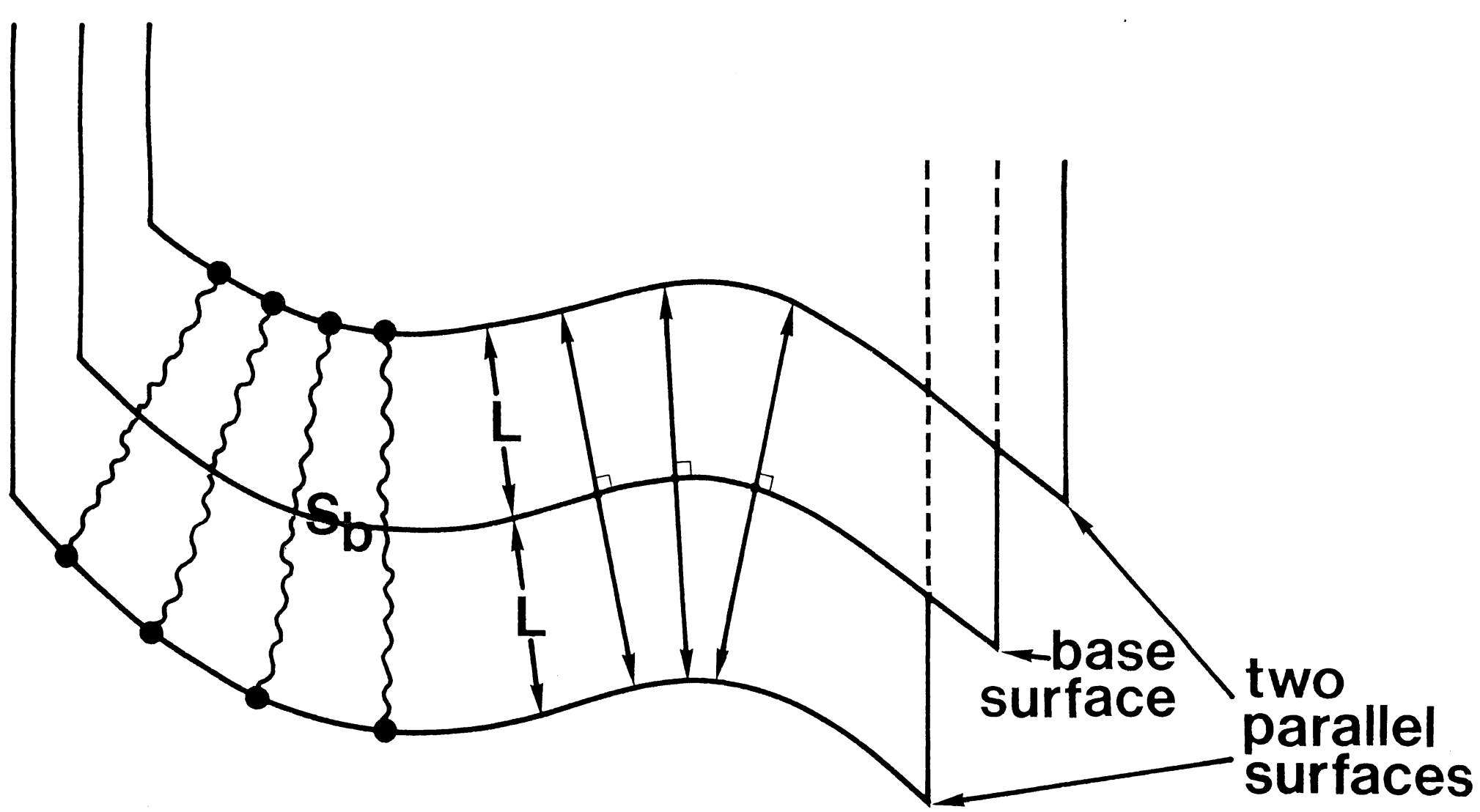

Figure 1. Schematic cross section of a bilayer with a midsurface, or "base" surface, at the center and two displaced surfaces assumed to represent the polar-apolar interface. Each point on the displaced surface is at a fixed distance along the normal to a point on the base surface. (Reprinted from ref. 18).

The mean curvatures $H_{1}$ and $H_{2}$ at the displaced surfaces are related to the mean $H_{b}$ and Gaussian $K_{b}$ curvatures of the base surface through

$$
\begin{aligned}
H_{1} & =\left(-H_{b}+L K_{b}\right) / N_{1} ; \quad H_{2}=\left(H_{b}+L K_{b}\right) / N_{2} ; \\
N_{1,2} & =1 \mp 2 H_{b} L+K_{b} L^{2}
\end{aligned}
$$

where it is essential to have a consistent sign convention; the positive normal to the base surface pointing towards displaced surface 2 . For the difference curvature we only consider the highest order term so that

$$
\Delta c_{1}=-\Delta c_{b} ; \quad \Delta c_{2}=\Delta c_{b}
$$

Using the expansion eq. (10) and allowing the two monolayers to be different we find for a bilayer

$$
g_{c}=a_{1} N_{1}\left[\left(-H_{b}+L K_{b}\right) / N_{1}-c_{01}\right]^{2}+a_{2} N_{2}\left[\left(H_{b}+L K_{b}\right) / N_{2}-c_{02}\right]^{2}+\left(b_{1}+b_{2}\right)\left(H_{b}^{2}-K_{b}\right)
$$


The extra factors $N_{1}$ and $N_{2}$ in the first two terms take into account the change in area going from the displaced surface to the base surface

$$
d A_{i}=N_{i} d A_{b}
$$

In the difference curvature term this higher order correction is neglected. Expanding eq. (14) including terms up to second order in the curvatures yields

$$
\begin{aligned}
g_{c} & =\left(a_{1}+a_{2}+b_{1}+b_{2}+2 a_{1} L^{2} c_{01}^{2}+2 a_{2} L^{2} d_{02}^{2}\right)\left(H_{b}-H_{b 0}\right)^{2} \\
& -\left(b_{1}+b_{2}+2 a_{1} c_{01} L+2 a_{2} c_{02} L+a_{1} c_{01}^{2} L^{2}+a_{2} c_{02}^{2} L^{2}\right) K_{b}+a_{1} c_{01}^{2}+a_{2} c_{02}^{2} \\
& -\left(c_{01} a_{1}-c_{02} a_{2}+c_{10}^{2} L a_{1}-c_{20}^{2} L a_{2}\right) H_{b 0}+\text { higher order terms }
\end{aligned}
$$

where the spontaneous bilayer mean curvature is

$$
H_{0 b}=\frac{c_{01} a_{1}-c_{02} a_{2}+c_{10}^{2} L a_{1}-c_{20}^{2} L a_{2}}{a_{1}+a_{2}+b_{1}+b_{2}+2 a_{1} L^{2} c_{01}^{2}+2 a_{2} L^{2} c_{02}^{2}}
$$

The spontaneous bilayer mean curvature can thus be nonzero if there is a difference in the two bending constants $a_{1}$ and $a_{2}$, or the two spontaneous monolayer mean curvatures, or both. For example, in certain cell membranes there are, e.g., proteins or macromolecules which are incorporated preferentially into one side of the bilayer, making the local symmetry $C_{\infty}$ rather than $D_{\infty}$. An example of this which is particularly pertinent with respect to our discussions of liquid crystals is the prolamellar body (PLB) of certain plant plastids, which are bicontinuous cubic phases in which the "inside", continuous with the cell cytoplasm, and "outside", continuous with the exterior of the cell, are not equivalent. Thus there is a nonzero mean curvature to the midsurface of the bilayer, in contrast to binary amphiphile/water cubic phases in which this bilayer mean curvature $H_{0 b}$ is zero by symmetry ${ }^{25}$.

For a symmetric bilayer $\left(a_{1}=a_{2}=a ; b_{1}=b_{2}=b ; c_{01}=c_{02}=c_{0}\right)$ eq. (16) simplifies to (to second order)

$$
\begin{aligned}
g_{c} & =2\left(a+b+2 a L^{2} c_{0}^{2}\right) H_{b}^{2}-2\left(b+2 a c_{0} L+a c_{0}^{2} L^{2}\right) K_{b}+2 a c_{0}^{2} \\
& =2 a\left(1-L c_{0}\right)^{2} H_{b}^{2}+2\left(b+2 a c_{0} L+a c_{0}^{2} L^{2}\right) \Delta c_{b}^{2}+2 a c_{0} 2
\end{aligned}
$$

Thus by imposing the bilayer symmetry we regain the form of eqs. (3) and (8) with explicit expressions for the coefficients in terms of those of the constituent monolayer. The bending rigidity $\kappa$ is found to be slightly larger than the sum over the two monolayer rigidities while the symmetric bending constant

$$
a_{b}=2 a\left(1-L c_{0}\right)^{2}
$$


can be either larger or smaller than the sum over the individual layers depending on the sign of $c_{0}$. For the bilayer to be stable relative to other structures $\left|L c_{0}\right|<1$ so that $a_{b}$ cannot reach very low values. Even more significant is that the saddle splay constant

$$
\bar{\kappa}_{b}=-b_{b}=-2\left(b+2 a c_{0} L+a c_{0}^{2} L^{2}\right)
$$

can actually change sign if a $\gg b>0$ which we will argue in the following section is typically the case. Then when $c_{0}<0, \bar{\kappa}_{b}$ can be positive and $b_{b}$ consequently negative. This change of sign of $\bar{\kappa}_{b}$ for negative values of $c_{0}$ is viewed in the $H_{b}, K_{b}$ approach as the source of the formation of the $L_{3}$ phase. Under such circumstances the bilayer prefers to form highly connected structures to achieve a slight curvature towards the solvent ${ }^{18}$.

The fact that the conventional saddle splay constant $\bar{\kappa}_{b}$ depends on the spontaneous monolayer mean curvature $c_{0}$, means that this "constant" is considerably more elusive than is suggested by the simple expression (3). This would plague the interpretation of experiments designed to measure $\bar{\kappa}_{b}$, and in fact one should expect $\bar{\kappa}_{b}$ to vary strongly when intensive parameters are changing. Since the whole point of introducing curvatures and associated constants is to attempt to interpret and extrapolate experimental results, the strongly varying nature of $\bar{\kappa}_{b}$ would seem to limit its usefulness, despite one attractive feature of expression (4) from a theoretical point of view due to the Gauss-Bonnet theorem.

The exception to this would be the case in which the bilayer spontaneous mean curvature $H_{b 0}$ is significantly nonzero, so that the curvature of the asymmetric bilayer as a whole would dominate the picture. However, even in such a case it might prove more insightful, though computationally more involved, to use the monolayer approach with $c_{01} \neq c_{02}$. Charvolin and Sadoc ${ }^{23}$ have shown elegantly that in the case of binary surfactant/water bicontinuous cubic phases, the symmetry of the bilayer leads to zero bilayer mean curvature, and indeed minimal surfaces provide models for the base surfaces of these structures.

Equation (12) must be recognized as occupying a central role in the discussion of curvature expressions. We argue herein that it is the mean curvature $H_{i}$ over the polarapolar interface that is physically most significant, and in the next section that therefore $b \ll a$ generally and the first term in expression (10) - with the curvatures measured at the polar-apolar interface - is dominant. On the other hand, equation (12) for $H_{i}$ in terms of the base surface requires both the mean and the Gaussian curvatures of the base surface, this means that in the bilayer approach, when the bilayer thickness cannot be neglected, using equation (6) both terms are of comparable magnitude. We maintain that the expansion (10) is the unique decomposition of the energies which results in two terms one of which is inherently smaller in magnitude. Furthermore, through eq. (14) for example, $c_{01}$ and $c_{02}$ are emphasized explicitly rather than suppressed. Refs. 18 and 19 show two of several cases where a more profound insight into the behaviour of bilayer systems can be gained by looking at the system in terms of the constituent monolayers. In understanding the relation between molecular properties and elastic constants much can 
be gained by referring to the general knowledge about surfactant systems that has been accumulated.

Surfactant Monolayer Free Energies and Curvature. During his extensive studies of surfactant phase equilibria Winsor ${ }^{15}$ found that the trends could be rationalized in terms of regular variations in the radius of the surfactant aggregate and he introduced a so scaled $R$-value. In the notation of this paper the Winsor $R$-value is simply $1 / c_{0}$ where $c_{0}$ is the preferred mean curvature of the surfactant monolayer. Note that $R$ is related to the preferred curvature $c_{0}$ rather than the spontaneous curvature $H_{0}$. The usefulness of the Winsor approach shows that $c_{0}$ is one of the most basic properties of a monolayer and it determines to a large extend the geometry of the aggregates found in a particular system.

Another approach to understanding the behaviour of surfactant systems was pioneered by $\operatorname{Tartar}^{10}$, revived by Tanford ${ }^{11}$, and further developed by Israelachvili and coworkers $^{12-14}$. The basic idea is to relate the packing requirement to fill space with the molecular shape as characterized by volume $v$, length $L_{0}$ and area of polar group $a_{0}$. The crucial dimensionless parameter is then $v /\left(L_{0} a_{0}\right)$ and for example when $v /\left(L_{0} a_{0}\right)=1$ a bilayer has optimal stability which is equivalent to $c_{0}=H_{0}=0$ in terms of curvature concepts. Note that the crucial area $a_{0}$ is that of the polar headgroup, which in the curvature approach translates to claiming that the curvature free energy is best associated with the polar-apolar interface. In terms of the approach to the curvature energies taken in the previous sections the dimensionless $v /\left(L_{0} a_{0}\right)$ ratio is related to $L_{0} c_{0}$ through

$$
v /\left(L_{0} a_{0}\right)=\left[1-L_{0} c_{0}+\left(L_{0} c_{0}\right)^{2} / 3\right]
$$

using spheres and spherical shells and

$$
v /\left(L_{0} a_{0}\right)=\left(1-L_{0} c_{0}\right)
$$

for cylinders and cylindrical shells. In general the bilayer half-thickness $L$ is close to the preferred value $L_{0}$. In this approach there is no local curvature term that corresponds to a Gaussian or difference curvature energy, although this could in principle be introduced. There are some fundamental differences between the $v / a_{0} L_{0}$ approach and the mean curvature approach making it difficult to compare the two unambiguously and independently of geometry; after all, $v / a_{0} L_{0}$ is dimensionless whereas $H$ has dimensions of inverse length, and even the two relations $(21 \mathrm{a}, \mathrm{b})$ are sufficient to demonstrate the geometry dependence of the relation between $v /\left(L_{0} a_{0}\right)$ and $c_{0}$ (or $\left.H_{0}\right)$.

Taking a more overall view, however, clearly in both the Winsor argument and in the packing approach, one has found it sufficient as a first approximation to only consider one parameter characterizing the monolayer. Furthermore, in both theories this one parameter is closely related to the preferred curvature $c_{0}$. The conclusion we draw is that the elastic constants $\{\kappa, \bar{\kappa}\}$ or $\{a, b\}$ are of lesser significance for the phase behaviour. The values of 
these constants become important in more free energy competitions between, for example, ternary lamellar phases and microemulsions, or between disordered $L_{3}$ phases and cubic phases, where detailed arguments involving entropy and interbilayer interactions must be given. Nevertheless experimental observations do allow some qualitative conclusions to be made concerning the signs and relative magnitudes of $a$ and $b$. For a monolayer in a liquid-like state all experience shows that both $a$ and $\kappa$ are positive. This is an explicit assumption in the theories presented above, which assumes the existence of an optimal mean curvature. The fact that the one parameter theories do so well indicates that $|b|$ is much smaller than $a$. It is generally found that hexagonal phases, normal and reversed, occur in a regular way between micellar and lamellar liquid crystalline phases. If $|b|$ were sizable it would have the effect of stabilizing the hexagonal phases, $b \ll 0$, or suppressing them, $b \gg 0$. Thus the difference curvature term seems to give only relatively small contributions to the curvature free energy of a monolayer. Concerning the sign of $b$, we are not aware of any conclusive evidence, but the intrinsic stability of lamellar phases ${ }^{24}$ indicates that in fact $b>0$ for a monolayer. As shown in the previous section the story can be different for a bilayer. That $b>0$ is also expected on theoretical grounds since it is generally, but not exclusively, the case that all other things being equal the higher the symmetry the lower the free energy in a disordered system.

Only with symmetry-breaking can $b$, as defined above, be negative. In that case, one has two choices. First, one can take $b<0$ with the same defining relation, but then higher order terms must of course be taken. The second alternative is to introduce a spontaneous $\Delta c_{0}$ into the difference curvature energy term.

In the case where a higher-order expansion is necessary, terms may be considered which involve the gradients of the curvatures. In such a case the Mainardi-Codazzi relations can be used to compute the gradients of the principle curvatures $c_{1}$ and $c_{2}$; see reference 18 for the appropriate formulae. Interestingly, if one is predisposed to the $H, K$ description, one finds that even in the formula for the gradient of the Gaussian curvature of a surface of constant mean curvature the factor $\left(c_{1}-c_{2}\right)^{2}$ appears - once again the fundamental symmetries of the curvatures are evidencing themselves.

Just before the submission of this paper, the authors were made aware of another treatment of curvature free energies by Fogden et. al which is related to, but differs from, the present approach. For now we choose only to say that, through the analysis of microscopic models as well as with stability arguments, those authors also concluded that the traditional expansion in terms of $H$ and $K$ is wrought with inherent difficulties. One common insight appears to be that simple expressions in the principle curvatures, such as the difference curvature which is so intuitively clear and physically meaningful, can only be clumsily written - non-analytically, in fact - in terms of $H$ and $K$ : thus $\Delta c=\sqrt{\left(H^{2}-K\right)}$, and $c_{i}=2 H \pm \sqrt{\left(H^{2}-K\right)}$ for the lowly principle curvatures themselves. 
Vesicle Stability. As an illustration of the usefulness of the concepts presented in previous sections of this paper we turn the attention to the question of the thermodynamic stability of unilamellar spherical vesicles. This is a question that has been extensively debated in the literature ${ }^{8,14,25,26}$ and due to the inherent possible metastability of vesicles it is very difficult to unequivocally experimentally establish thermodynamic stability. Such a stability has only been claimed in a few cases ${ }^{27-30}$ and clearly a theoretical analysis of the problem can help to explain why this is a rare event.

In ref. 18 it was shown that under the circumstances when the monolayer preferred curvature is negative, $c_{0}<0$, i.e. curvature towards solvent is preferred, a multiply connected bilayer can be formed. The formation of vesicles represent the opposite trend where the mean curvature $c>0$ and one could expect spontaneous vesiculization in some regime when monolayer $c_{0}>0$. This is an argument often presented, albeit often expressed in terms of the packing parameter $v / a_{0} L_{0}^{13,14,31}$. As we will demonstrate below this reasoning point in the right direction but the problem is more involved. Among other aspects we will demonstrate that when it comes to finer balances a one-parameter theory involving, for example, only $v / a_{0} L_{0}$, is too simplified. This is particularly true when one is dealing with structures that are not locally homogeneous. In the vesicle problem there is an interesting and important inhomogeniety between the inside and the outside monolayers.

Using the decomposition of energies (eqn. (6)) in $H$ and $K$ is cumbersome even in the case of spherical vesicles since $\bar{\kappa} \neq 0$ in general, and the Gaussian curvature energy term per molecule depends on the aggregation number in the vesicle - as well as on the value of $\bar{\kappa}$. On the other hand, the decomposition using $H$ and $\Delta c$ is very straightforward, and is particularly advantageous in the case treated below where a higher order expansion is needed. Thus, if we restrict the discussion to spherical vesicles the difference curvature $\Delta c=0$ and a straightforward application of eq. (8) gives the curvature free energy to second order as

$$
G_{c}=4 \pi R_{2}^{2} a\left(1 / R_{2}-c_{0}\right)^{2}+4 \pi R_{1}^{2} a\left(-1 / R_{1}-c_{0}\right)^{2}
$$

where the inner (1) and outer (2) monolayers have been assumed chemically equivalent. If eq. (22) is expanded for $1 / R_{i} \rightarrow 0$ with a bilayer thickness of $2 L$ we regain the factor $2 a\left(1-L c_{0}\right)^{2}$ of eq. (19). In discussing smaller size vesicles it is important to specify the constraints on area and volume. For an incompressible fluid, molecular volume $v$, and a constant area per polar group $\left(a_{0}\right)$, the relation between the radii $R_{2}$ and $R_{1}$ follows from

$$
\begin{aligned}
& N v_{0}=\left(R_{2}^{3}-R_{1}^{3}\right) 4 \pi / 3 \\
& N a_{0}=4 \pi\left(R_{2}^{2}+R_{1}^{2}\right)
\end{aligned}
$$

where $N$ is the number of molecules in the vesicle. The free energy per unit area is

$$
\frac{G}{N a_{0}}=2 a \frac{\left[1-c_{0}\left(R_{2}-R_{1}\right)\right]}{R_{1}^{2}+R_{2}^{2}}+a c_{0}^{2}
$$


under the constraint

$$
R_{2}^{3}-R_{1}^{3}-3 L\left(R_{2}^{2}+R_{1}^{2}\right)=0
$$

where $L=v / a_{0}$ is the monolayer thickness in the planar configuration. In this state $R_{1} \rightarrow R_{2} \rightarrow \infty ; R_{2}-R_{1}=2 L$ and $G / N a_{0} \rightarrow a c_{0}^{2}$. To obtain a lower free energy for vesicles $c_{0}\left(R_{2}-R_{1}\right)>1$. The constraint eq. (25) implies that $R_{2}-R_{1}$ varies from $2 L$ to $3 L$ as $R_{1}$ decreases to zero. Thus, in the given approximation, vesicles could only be stable relative to a planar state when $c_{0} L>1 / 3$. However, for radii $R_{2}$ approaching $3 L$ the approximations in the second order curvature analysis are no longer realistic. Furthermore for $c_{0} L$ as large as $1 / 3$ a cylindrical aggregate is more favourable than the vesicle, assuming $|b| \ll a$. The conclusion being that within the second order curvature analysis vesicles are unstable. Helfrich and Harbich8 have recently presented a regime where vesicles are found unstable. This is when $\kappa-2 \bar{\kappa}<0$ which in the present notation implies $a_{b}<0$. According to eq. (19) combined with the observation that $a>0, a_{b} \geq 0$ always. Although related to Helfrich and Harbich criterion, the description in eqs. (24) and (25) contains more detail and should be more precise. The analysis supports the qualitative conclusion that vesicles are most likely to be stable $c_{0}>0$ but in contrast to the results of packing arguments the effect does not come out strong enough to actually support the notion of vesicle stability. However, in the second order treatment of curvature energies the vesicles are only marginally unstable, which could indicate that in a more elaborate description relative free energies could shift sufficiently to give stable vesicles.

In a small vesicle the radii of curvature on the inside and the outside are markedly different and one is no longer necessarily dealing with small deviations from the preferred curvature. It is then appropriate to consider higher order terms in the expansion. For spherical vesicles, $\Delta c=0$, a fourth order expansion of the monolayer free energy density gives

$$
g=a_{2}\left(H-c_{0}\right)^{2}+a_{3}\left(H-c_{0}\right)^{3}+a_{4}\left(H-c_{0}\right)^{4}
$$

Here $a_{2}$ and $a_{4}>0$ while the sign of $a_{3}$ determines the orientation of the skewness of $g$ versus $H$. Such higher order terms have been envoked to explain the minimum size of vesicles $^{32}$. Note the simplicity of obtaining the fourth order expansion when the difference curvature formalism is followed; if the Gaussian curvature formalism were used instead, terms such as $\left(H-H_{0}\right)^{2} K, K^{2}$, etc. would be encountered.

Using eq. (26) we could follow the same procedure as with the second order expansion and deduce the free energy per unit area which should be minimized under the constraint eq. (25). This is straightforward but tedious and instead we make a stability analysis of the planar state. The mean curvatures of the two monolayers are related to the curvature of the midplane through eqs. (12a-c), where $K_{b}=H_{b}^{2}$ so that $N_{1,2}=\left(1+H_{b} L\right)^{2}$. Inserting 
this into eq. (26) and correcting for the change in area element gives for the bilayer

$$
\begin{gathered}
g=2\left(a_{2} c_{0}^{2}-a_{3} c_{0}^{3}+a_{4} c_{0}^{4}\right)+2 H_{b}^{2}\left\{a_{2}\left(1-c_{0} L\right)^{2}-a_{3} c_{0}\left(3-3 c_{0} L+c_{0}^{2} L^{2}\right)\right. \\
\left.+a_{4} c_{0}^{2}\left(6-4 c_{0} L+c_{0}^{2} L^{2}\right)\right\}+ \\
+2 H_{b}^{4}\left\{a_{4}\left(1-4 c_{0} L\right)-a_{3} L\right\}+\text { higher order terms }
\end{gathered}
$$

The second order term can be negative if, for $c_{0}>0, a_{3}$ is sufficiently large and positive. A large value of $c_{0}$ also make a negative value more likely, provided that the fourth order coefficient is small. The range for a negative value of the $H^{2}$ term is approximately given by $3 a_{3} c_{0} \geq a_{2}$ while $2 a_{4} c_{0} \ll a_{3}$. Under these circumstances a bilayer will bend spontaneously to a vesicle of an optimal radius that can be determined by analyzing the complete expression in eq. (27). The instability of the planar configuration arises since the cost of forming the negative curvature inner monolayer is less unfavourable than suggested by the quadratic expansion. However, the conditions under which vesicles would be stable seem rather restricted and it seems unlikely event. A search for a global minimum, rather than the stability analysis given above, would give the same qualitative picture but with somewhat less stringent conditions on the elastic constants for vesicle stability.

Another mechanism that can act to facilitate vesicle stability is operating in a system of mixed amphiphiles. Normally the preferred curvature is a function of composition and at the formation of a vesicle there is a possibility of a partial segregation of the components between the inner and outer monolayers. This effect has been analyzed by Leibler in general terms $^{33}$. In a second order analysis the combined curvature and chemical monolayer free energy density can be written

$$
g\left(H_{1} X\right)=a\left\{H-c_{0}(X)\right\}^{2}+g(X)
$$

where $g(X)$ is the bulk free energy per unit area of a binary mixture with mole fraction $X$ of component 2.

Both the entropy of mixing and the molecular interactions contribute to $g(X)$. We will see that the crucial term entering the vesicle stability is the second derivative $d^{2} g / d X^{2}$, which for a miscible system must be positive. The entropy term gives a contribution $R T[1 / X+1 /(1-X)]$, assuming random mixing. This term can be partly cancelled by an interaction term depending on the detailed chemistry.

For the composition dependence of the preferred curvature we make the simplest possible assumption that it varies linearly with composition

$$
c_{0}(X)=X\left(c_{20}-c_{10}\right)+c_{10}
$$

where $c_{20}$ and $c_{10}$ are the preferred curvatures for the pure components. When a vesicle is formed the total composition $X$ is given (assuming homogeniety in the system) but the individual monolayers can exchange material so that

$$
X_{2}=X+\Delta X_{2} \text { and } X_{1}=X+\Delta X_{1}
$$


where the indices refer to the two monolayers. If we assume, for simplicity, that the two components have the same volumes $v$ and areas $a_{0}$, and if the expansion is made around $H_{b}=0$ then $\Delta X_{2}=-\Delta X_{1}=\Delta X$. the free energy per unit area of the vesicle is

$$
g\left(H_{b}, \Delta X\right)=2 a\left\{H_{b}^{2}\left(1-L c_{0}(X)\right)^{2}+c_{0}^{2}(X)-2 H_{b} \Delta c_{0} \Delta X+\Delta c_{0}^{2} \Delta X^{2}\right\}+\Delta X^{2} \frac{d^{2} g}{d X^{2}}
$$

where $\Delta c_{0}=c_{20}-c_{10}$. Define $g\left(H_{b}\right)$ through

$$
g\left(H_{b}, \Delta X\right) ; \quad \partial g / \partial \Delta X=0
$$

Minimizing $g\left(H_{b}, \Delta X\right)$ with respect to $\Delta X$ yields

$$
\Delta X=2 a \Delta c_{0} H_{b} /\left(2 a \Delta c_{0}^{2}+\frac{d^{2} g}{d X^{2}}\right)=\Delta c_{0} H_{b} / N
$$

The coefficient for the second order term is renormalized from $2 a\left(1-L c_{0}\right)^{2}$ to

$$
2 a_{2}\left(1-2 L c_{0}+L^{2} c_{0}^{2}-4 \Delta c_{0}^{2} / N+2 \Delta c_{0}^{4} / N^{2}+\Delta c_{0}^{2} d^{2} g / d X^{2} / N^{2}\right)
$$

for a stable mixture $d^{2} g / d X^{2}>0$ and when $d^{2} g / d X^{2} \gg \Delta c_{0}^{2}$ the correction terms are negligible, while for $d^{2} g / d X^{2} \ll \Delta c_{0}^{2}$ the coefficient reduces to $2 a\left(-2 L c_{0}+L^{2} c_{0}^{2}\right)$. If higher order terms in $H_{b}$ are included in the relation between $\Delta X_{1}$ and $\Delta X_{2}$ the second order term reduces to zero. This shows by way of example how the extra degree of freedom introduced by having two amphiphilic components could greatly facilitate the spontaneous formation of vesicles. This occurs when the decrease in entropy associated with having different concentrations in the inner and outer monolayers is partly compensated by an interaction term reducing the value of $d^{2} g / d X^{2}$.

In the presentation of the vesicle problem we have left out the role of interbilayer forces such as double layer, van der Waals and hydration forces. Strong forces either attractive or repulsive will favour the most ordered arrangement of the bilayers, which is the lamellar phase. For a non-charged system the attractive van der Waals force will tend to produce stacked bilayers more or less irrespective of concentration. For sugar lipids and phosphatidylethanolamine attractions are stronger than for phosphatidylcholine. For charged amphiphiles, like the dialkyldimethylammonium systems, studied by Evans, Ninham and coworkers ${ }^{27}$, the repulsive intervesicular force can be diminished by dilution but there is always an intravesicular contribution which tends to destabilize the vesicles. The role of such long range force cannot satisfactorily be incorporated into a curvature free energy due to the additivity assumption inherent in eq. (4). We have furthermore neglected the role of the entropy increase associated with the formation of finite size vesicles from infinite sheets. this entropic effect is partly offset by the entropy of the bending indulations 
of the infinite bilayer. A complete analysis of the problem of vesicle stability would have to include these factors, in addition to the bending elastic energies, which we, however, consider to be the most important factor.

During completion of this paper, an analysis of the stability of spherical vesicles by Safran et al. $^{34}$ appeared. The basic approach to the vesicle stability problem in ref. 34 is much the same as that presented above. These authors have concentrated on the twocomponent vesicle problem having a more molecular description of the surfactant mixture.

Acknowledgements. H. Wennerström wishes to thank the Department of Chemical Engineering and Material Science at the University of Minnesota for a George T. Percy distinguished visiting professorship, 1988-1989 during which time a major part of this work was performed. D. Anderson gratefully acknowledges a postdoctoral grant from the Swedish Science Research Council.

\section{REFERENCES}

[1] Helfrich, W.Z., Naturforsch, 28c (1973), p. 693.

[2] Note that many authors, including Helfrich, do not use the factor $1 / 2$ in the definition of $H$. In this paper we use the convention of the mathematical literature. Because of these differences, factors of 2 may be needed to put our formulae in exact correspondence with formulae used in other publications.

[3] Due to the difference in the definition of $H$ a factor of 2 (two) appears in the numerator, preserving the conventional definition of $\kappa$.

[4] Struik, D.J., Lectures on classical differential geometry, Addison Wesley, Cambridge, Mass. 1980.

[5] Bessis, M., Living blood cells and their ultrastructure, Springer, Berlin 1973.

[6] Survuss, R.M.; Harbich, W.; Helfrich, W., Biochim. Biophys., Acta 436 (1976), p. 900.

[7] Helfrich, W.; Servuss, R.M., Nuovo Cimento, 30 (1984), p. 137.

[8] Helfrich, W.; HaRbich, W., in "Physics of Amphiphilic Layers", eds. Meunier, J.; Langevin, D.; Boccara, N. Springer, Berlin (1987), p. 58.

[9] See for example Safran, S.; Roux, d.; Milner, S.T.; Cates, M.E.; Andelman, D. in Physics of Amphiphilic Layers, eds. Meunier, J.; Langevin, D.; Boccara, N. Springer, Berlin (1987), p. 291.

[10] Tartar, H.V.J., Phys. Chem., 59 (1955), p. 1195.

[11] Tanford, C., The hydrophobic effect, Wiley, New York (1973).

[12] Israelachvidi, J.N.; Mitchel, D.J., Ninham, B.W.J., Soc. Faraday Trans. I , 72 (1976), p. 1525.

[13] Israelachvili, J.N.; Marcelja, s.; Horn, R.Q., Rev. Biophys. , 13 (1980), p. 121.

[14] Israelachvili, J.N., in "Surfactants in Solutions", eds. Mittal, K.L.; Bothorel, P. Plenum Press Vol. 4 (1987), p. 3.

[15] Winsor, P., Chem. Rev., 68 (1968), p. 1.

[16] Tiddy, G.J.T., Presentation at "International Workshop on Supermolecular Fluids", Amsterdam, Oct. 24-26, 1990.

[17] Auvray, X., Petipas, C., Perche, T., Anthore, R., Marti, M.J., Rico, I., Lattes, A.J., Phys. Chem., 94 (1990), p. 8604.

[18] Anderson, D.; Wennerström, H.; Olsson, U.J., Phys. Chem. , 93 (1989), p. 4532.

[19] Ström, P., ANDERson, D., Langmuir, (submitted for publication).

[20] Duplantier, B., Physica A, 168, p. 179.

[21] Kirk, G.L.; Gruner, S.M.; Stein, D.L., Biochem, 23 (1984), p. 1093.

[22] Anderson, D.M.; Gruner, S.M.; Leibler, S., Proc. Nat. Acad. Sci. (USA), 85 (1988), p. 5364.

[23] Sadoc, J.F.; Charvolin, J., J. Phys., 48 (1987), p. 1559.

[24] Larché, F.C.; Appell, J.; Porte, G.; Bassereau, P.; Marignan,, J. Phys. Rev. Lett., 56 (1986), p. 1700 . 
[25] Suezaki, Y.; TSujI, N.J., colloid Interface Sci., (1986), p. 131.

[26] Benton, W.J., in "Physics of Amphiphilic Layers", eds. Meunier, J.; Langevin, D.; Boccara, N. Springer, Berlin (1987), p. 207.

[27] Talmon, Y.; Evans, D.F.; Ninham, B.W., Science, 221 (1983), p. 1047.

[28] Hauser, H.; Gains, N.; Müller, M., Biochemistry, 22 (1983), p. 4775.

[29] Almog, S.; Kushir, T.; Nir, S.; Lichtenberg, D., Science, 25 (1986), p. 6597.

[30] Kaler, E.W.; Murthy, A.K.; Rodrigueq, J.A.; Zasadzinski, Science, 245 (1989), p. 1371.

[31] Evans, D.F., Langmuir, 4 (1988), p. 3.

[32] Helfrich, W.J., Physique, 47 (1986), p. 321.

[33] Leibler, S.J., Physique, 47 (1986), p. 507.

[34] Safran, S.A.; Pincus, P.; Andelman, D., Science, 248 (1990), p. 354. 


\section{Recent IMA Preprints}

Title

Title

Avner Friedman and Bei Hu, The Stefan problem with kinetic condition at the free boundary

M.A. Grinfeld, The stress driven instabilities in crystals: mathematical models and physical manifestations

Bei Hu and Lihe Wang, A free boundary problem arising in electrophotography: solutions with connected toner region

Yongzhi Xu, T. Craig Poling, and Trent Brundage, Direct and inverse scattering of time harmonic acoustic waves in an inhomogeneous shallow ocean

Steven J. Altschuler, Singularities of the curve shrinking flow for space curves

Steven J. Altschuler and Matthew A. Grayson, Shortening space curves and flow through singularities

Tong Li, On the Riemann problem of a combustion model

L.A. Peletier \& W.C. Troy, Self-similar solutions for diffusion in semiconductors

C.J. van Duijn, L.A. Peletier \& R.J. Schotting, On the analysis of brine transport in porous media

Minkyu Kwak, Finite dimensional description of convective reaction-diffusion equations

Minkyu Kwak, Finite dimensional inertial forms for the 2D Navier-Stokes equations

Victor A. Galaktionov and Sergey A. Posashkov, On some monotonicity in time properties for a quasilinear parabolic equation with source

Victor A. Galaktionov, Remark on the fast diffusion equation in a ball

Hi Jun Choe and Lihe Wang, A regularity theory for degenerate vector valued variational inequalities

Vladimir I. Oliker and Nina N. Uraltseva, Evolution of nonparametric surfaces with speed depending on curvature, II. The mean curvature case.

S. Kamin and W. Liu, Large time behavior of a nonlinear diffusion equation with a source

Shoshana Kamin and Juan Luis Vazquez, Singular solutions of some nonlinear parabolic equations

Bernhard Kawohl and Robert Kersner, On degenerate diffusion with very strong absorption

Avner Friedman and Fernando Reitich, Parameter identification in reaction-diffusion models

E.G. Kalnins, H.L. Manocha and Willard Miller, Jr., Models of $q$-algebra representations I. Tensor products of special unitary and oscillator algebras

Robert J. Sacker and George R. Sell, Dichotomies for linear evolutionary equations in Banach spaces

Oscar P. Bruno and Fernando Reitich, Numerical solution of diffraction problems: a method of variation of boundaries

Oscar P. Bruno and Fernando Reitich, Solution of a boundary value problem for Helmholtz equation via variation of the boundary into the complex domain

Victor A. Galaktionov and Juan L. Vazquez, Asymptotic behaviour for an equation of superslow diffusion. The Cauchy problem

Josephus Hulshof and Juan Luis Vazquez, The Dipole solution for the porous medium equation in several space dimensions

Shoshana Kamin and Juan Luis Vazquez, The propagation of turbulent bursts

Miguel Escobedo, Juan Luis Vazquez and Enrike Zuazua, Source-type solutions and asymptotic behaviour for a diffusion-convection equation

Marco Biroli and Umberto Mosco, Discontinuous media and Dirichlet forms of diffusion type

Stathis Filippas and Jong-Shenq Guo, Quenching profiles for one-dimensional semilinear heat equations

H. Scott Dumas, A Nekhoroshev-like theory of classical particle channeling in perfect crystals

R. Natalini and A. Tesei, On a class of perturbed conservation laws

Paul K. Newton and Shinya Watanabe, The geometry of nonlinear Schrödinger standing waves

S.S. Sritharan, On the nonsmooth verification technique for the dynamic programming of viscous flow

Mario Taboada and Yuncheng You, Global attractor, inertial manifolds and stabilization of nonlinear damped beam equations

Shigeru Sakaguchi, Critical points of solutions to the obstacle problem in the plane

F. Abergel, D. Hilhorst and F. Issard-Roch, On a dissolution-growth problem with surface tension in the neighborhood of a stationary solution

Erasmus Langer, Numerical simulation of MOS transistors

Haïm Brezis and Shoshana Kamin, Sublinear elliptic equations in $\mathbb{R}^{n}$

Johannes C.C. Nitsche, Boundary value problems for variational integrals involving surface curvatures

Chao-Nien Chen, Multiple solutions for a semilinear elliptic equation on $\mathbb{R}^{N}$ with nonlinear dependence on the gradient

D. Brochet, X. Chen and D. Hilhorst, Finite dimensional exponential attractor for the phase field model

Joseph D. Fehribach, Mullins-Sekerka stability analysis for melting-freezing waves in helium-4

Walter Schempp, Quantum holography and neurocomputer architectures

D.V. Anosov, An introduction to Hilbert's 21st problem

Herbert E Huppert and M Grae Worster, Vigorous motions in magma chambers and lava lakes 
Robert L. Pego and Michael I. Weinstein, A class of eigenvalue problems, with applications to instability of solitary waves

Mahmoud Affouf, Numerical study of a singular system of conservation laws arising in enhanced oil reservoirs

Darin Beigie, Anthony Leonard and Stephen Wiggins, The dynamics associated with the chaotic of tangles two dimensional quasiperiodic vector fields: theory and applications

866

867

868

869

870

871

872

873

874

875

876

877

878

879

880

881

882

883

884

885

886

887

888

889

890

891

892

893

894

895

896

897

898

899

900

901

902

903

904

905

906

907

908

909

910

911

Gui-Qiang Chen and Tai-Ping Liu, Zero relaxation and dissipation limits for hyperbolic conservation laws

Gui-Qiang Chen and Jian-Guo Liu, Convergence of second-order schemes for isentropic gas dynamics

Aleksander M. Simon and Zbigniew J. Grzywna, On the Larché-Cahn theory for stress-induced diffusion

Jerzy Łuczka, Adam Gadomski and Zbigniew J. Grzywna, Growth driven by diffusion

Mitchell Luskin and Tsorng-Whay Pan, Nonplanar shear flows for nonaligning nematic liquid crystals

Mahmoud Affouf, Unique global solutions of initial-boundary value problems for thermodynamic phase transitions

Richard A. Brualdi, Keith L. Chavey and Bryan L. Shader, Rectangular $L$-matrices

Xinfu Chen, Avner Friedman and Bei Hu, The thermistor problem with zero-one conductivity II

Raoul LePage, Controlling a diffusion toward a large goal and the Kelly principle

Raoul LePage, Controlling for optimum growth with time dependent returns

Marc Hallin and Madan L. Puri, Rank tests for time series analysis a survey

V.A. Solonnikov, Solvability of an evolution problem of thermocapillary convection in an infinite time interval

Horia I. Ene and Bogdan Vernescu, Viscosity dependent behaviour of viscoelastic porous media

Kaushik Bhattacharya, Self-accommodation in martensite

D. Lewis, T. Ratiu, J.C. Simo and J.E. Marsden, The heavy top: a geometric treatment

Leonid V. Kalachev, Some applications of asymptotic methods in semiconductor device modeling

David C. Dobson, Phase reconstruction via nonlinear least-squares

Patricio Aviles and Yoshikazu Giga, Minimal currents, geodesics and relaxation of variational integrals on mappings of bounded variation

Patricio Aviles and Yoshikazu Giga, Partial regularity of least gradient mappings

Charles R. Johnson and Michael Lundquist, Operator matrices with chordal inverse patterns

B.J. Bayly, Infinitely conducting dynamos and other horrible eigenproblems

Charles M. Elliott and Stefan Luckhaus, 'A generalised diffusion equation for phase separation of a multi-component mixture with interfacial free energy'

Christian Schmeiser and Andreas Unterreiter, The derivation of analytic device models by asymptotic methods

LeRoy B. Beasley and Norman J. Pullman, Linear operators that strongly preserve the index of imprimitivity

Jerry Donato, The Boltzmann equation with lie and cartan

Thomas R. Hoffend Jr., Peter Smereka and Roger J. Anderson, A method for resolving the laser induced local heating of moving magneto-optical recording media

E.G. Kalnins, Willard Miller, Jr. and Sanchita Mukherjee, Models of $q$-algebra representations: the group of plane motions

T.R. Hoffend Jr. and R.K. Kaul, Relativistic theory of superpotentials for a nonhomogeneous, spatially isotropic medium

Reinhold von Schwerin, Two metal deposition on a microdisk electrode

Vladimir I. Oliker and Nina N. Uraltseva, Evolution of nonparametric surfaces with speed depending on curvature, III. Some remarks on mean curvature and anisotropic flows

Wayne Barrett, Charles R. Johnson, Raphael Loewy and Tamir Shalom, Rank incrementation via diagonal perturbations

Thierry Dombre, Alain Pumir, and Eric D. Siggia, On the interface dynamics for convection in porous media Mingxiang Chen, Xu-Yan Chen and Jack K. Hale, Structural stability for time-periodic one-dimensional parabolic equations

Hong-Ming Yin, Global solutions of Maxwell's equations in an electromagnetic field with the temperaturedependent electrical conductivity

Robert Grone, Russell Merris and William Watkins, Laplacian unimodular equivalence of graphs

Miroslav Fiedler, Structure-ranks of matrices

Miroslav Fiedler, An estimate for the nonstochastic eigenvalues of doubly stochastic matrices

Miroslav Fiedler, Remarks on eigenvalues of Hankel matrices

Charles R. Johnson, D.D. Olesky, Michael Tsatsomeros and P. van den Driessche, Spectra with positive elementary symmetric functions

Pierre-Alain Gremaud, Thermal contraction as a free boundary problem

K.L. Cooke, Janos Turi and Gregg Turner, Stabilization of hybrid systems in the presence of feedback delays

Robert P. Gilbert and Yongzhi Xu, A numerical transmutation approach for underwater sound propagation

LeRoy B. Beasley, Richard A. Brualdi and Bryan L. Shader, Combinatorial orthogonality

Richard A. Brualdi and Bryan L. Shader, Strong hall matrices

Håkan Wennerström and David M. Anderson, Difference versus Gaussian curvature energies; monolayer versus

bilayer curvature energies applications to vesicle stability
Shmuel Friedland, Eigenvalues of almost skew symmetric matrices and tournament matrices 